\title{
Feature Level Fusion Algorithm for Iris and Face
}

Olatunji K. A. ${ }^{1}$, Oguntimilehin A. ${ }^{2}$,Aweh O. M. ${ }^{3}$,Adeyemo O. A. ${ }^{4}$

${ }^{1}$ Department of Mathematical and Physical Sciences

AfeBabalola University Ado Ekiti, Ekiti State, Nigeria olatunjika@abuad.edu.ng,

${ }^{2}$ Department of Mathematical and Physical Sciences

AfeBabalola University Ado Ekiti, Ekiti State, Nigeria abiodunoguns@abuad.edu.ng

${ }^{3}$ Department of Mathematical and Physical Sciences

AfeBabalola University Ado Ekiti, Ekiti State, Nigeria opaniaweh@gmail.com

${ }^{4}$ Department of Mathematical and Physical Sciences AfeBabalola University Ado Ekiti, Ekiti State, Nigeria adeyemo@abuad.edu.ng

Received Date : November 06, 2021 Accepted Date : November 28, 2021 Published Date : December 07, 2021

\begin{abstract}
Some Bi-modal or multimodal recognition systems do not contain rich information needed for identification because information supplied to the biometric classifier are consolidated oncethe conclusions of the matching algorithm have been acquired. Feature based Fusion algorithm has the distinction of having richer information due to the integration of the extracted information before the application of the classifiers. Support Vector Machine over time has shown its unbeatable classification of the biometrics characteristics over other supervised learning classifiers due to its ability to minimize the structural risk simultaneously with bound on the margin complexity and by being solved using a quadratic optimization problem. Neural Network in contrast is a non-parametric estimator which is robust to errors in the training data used for classification and regression.

Therefore in this research, algorithms for feature extraction of iris and face for recognition is designed; a recognition system using SVM and Multilayer Perceptron (MLP) is also designed based on the extracted features and the designed model is implemented using MATLAB.
\end{abstract}

Key words: Biometrics, Face, Iris, Gabor Filter, GLCM

\section{INTRODUCTION}

Biometric technology, that is grounded on sole physical and behavioural features of human body such as face, fingerprint, hand shapes, iris, palmprint, keystroke, signature, voice, also vein structure [12] is considered a substitute to prevailing methods in a pronounced deal of application areas such as bank Automatic Teller Machines (ATM), Telecommunications, Internet and Airport securities, human computer interaction/ human machine interface, or even driver's drowsiness detection system.

Face recognition has turn out to be a common research focus in the machine vision field and also one of the successful applications of image processing which plays a significant part in many today applications [11].Landmarks, or features from an image of the subject's face is extracted by some recognition systems to enable them identify faces. For example, the comparative position, size, and/or shape of the eyes, nose, cheekbones, and jaw of individual may be analyzed by an algorithm. The algorithm will now use these features to search for other images by checking the matching features. A gallery of face images are standardized by other algorithms and at that point compress the face data, the image that is useful for face detection will only be 
saved. The image that is reviewed is then compared with the face data. The template matching techniques which is one of the successful earliest models is applied to some of important facial features, thereby yielding a class of compacted face representation [10].

Iris patterns are distinctive and are gotten through video based image acquirement system. Each iris configuration is containing a complex pattern. This comprises of different kinds of attributes identified as corona, crypts, filaments, freckles, pits, furrows, striations and rings [15].

The integration or consolidation of multiple biometrics features for the accurate determination and verification of an identity is known as Biometric fusion [4]. The key purposes for biometric fusion are the improvement in the system's accuracy, efficiency, applicability, and robustness. Information fusions in biometric methods are classified into two categories: pre and post classification fusion. The combination of information prior to the application of any classifier or matching algorithm is refers to as Pre-classification fusion. While the combinations of information after the decisions of the classifiers have been obtained is known as post-classification fusion[13]. This information can be consolidated at either the sensor level or at the feature level before any classification or matching occurs. The feature sets of various characteristics are unified and a new feature set is produced at the feature level fusion; the new feature set is then used in the matching and decision- making modules of the biometric system.In this research pre-classification fusion is employed.

\section{LITERATURE REVIEWED}

Some of the researches reviewed are presented as follows:

[5] employed Support Vector Machines, PCA and LDA in Face Recognition. The implementation of an automated mechanism sustained systems that can identifies individuals in the dataset that were not trained before is the goal of the research (when the initialization and the training of the typical sample images has been carried out). In order to achieve the objectives of the research, the training images were done by projecting them unto both the Principal Component Analysis and Linear Discriminant Analysis subspace for the dimensionality reduction.
The individual dataset are also projected to the same subspace and related by different distance metrics between the image and training images for both methods. Distance metrics employed are Mahalinobis Cosine for PCA and LDASoft for LDA. Classification of nonlinear mapping unto higher dimensional feature space was done using Support Vector Machine. At the end of the experiments with FERET database the proposed methods PCA+SVM and LDA+SVM give an improved recognition rate than traditional PCA and LDA methods. Combination LDA+SVM also give better reliable results than LDA alone. Altogether, more than 300 tests were made and achieved maximum recognition rates near $100 \%$ (LDA+SVM once actually 100\%).

Iris recognition system using PCA based Dimensionality reduction and SVM is proposed by [16]. He realized that IRIS recognition difficult possibly will be well thought-out as challenges of categorizing the attributes removed from a test iris dataset to any of the attributes collections which are gotten as training images or iris image of identified classes or persons. The hamming distance based iris recognition problem is not as popular as the Feature classification based IRIS recognition techniques; because the distance based recognition system is not as good or efficient as the feature based classifiers. In this research, the proposed Daugman algorithm [3] for bringing out a salient huge numbers of features from iris image is employed. And then, PCA based linear dimensionality reduction procedure is used to moderate the features. The Gabor filter result which is the highest Eigen values extracted is employed as a feature vector as a training data for SVM classifier which classifies the largest Eigen values extracted from the test IRIS image. The iris dataset is obtained from CASIA and MMU database for the experimentation of the research. The result shows that the overall accuracy of the system is close to $99.6 \%$ on CASIA IRIS database and $97 \%$ on MMU database. The result and the effectiveness are better compared to those that have existed before.

[8]Compared Quality-Based Fusion of Face and Iris Biometrics. Q-FIRE database consisting of a multimodal data of face and iris biometrics taken at a well-defined controlled level was used. The FaceIT SDK was engaged for the production of face match scores. An improved version of the Masek open source software was employed to generate Iris match 
scores. Implementation of the score level fusion was done using the fixed sum rule. An independent training set of match scores were utilized for the second fusion method implementation of the likelihood ratio statistic modelled with Gaussian mixture models (GMM). In [6], a biometric authentication where iris and fingerprint features were combined together using score level fusion was proposed. Quantile transform of the real and impostor dataset; and a power transform which additionally enhances the score distribution to help linear classification were the two components methods employed. The scores were later normalized using the novel quantile power transform.

[2] researched on the role of artificial intelligence using various sensors for identification of person's iris. Neural Network models that can easily made decision were used and they are as follows: Multilayer perceptron (MLP), Radia Based Function Network (RBF), Self Organizing Map (SOM) and Support Vector Machine (SVM). [1] researched on learning and recognizing faces from motionless images to video sequences. A new methodology that is based on Local Binary Patterns (LBP) consisting of partitioning the facial image into minor compartments from which LBP features are mined and combined into a distinct feature histogram efficiently representing the face image was proposed by him. Then, a nearest neighbour classifier in the computed feature space with Chi-square as dissimilarity metric is used to carry out the face recognition. A frontal faces in complex gray scale images were detected using a trained second-degree polynomial kernel SVMclassifier.

[14]employed Adaptive MultiscaleRetinex and Support Vector Machine as a classifier to develop a Face identification System. They proposed SVM method for a face identification system using Yale and ORL databases which contained 165 images that has been converted to grayscale of 15 persons for Yale and 40 classes for ORL. Each subject consists of images, different facial expression or configuration including with and without glasses are included, different illumination variation and changes in facial expression. The images size was about $96 \times 106$, this image size is the cropped face images. Adaptive MultiscaleRetinex (AMSR) by [7] was applied on cropped images to lessen the illumination effects. Meanwhile, a face identification system needs multiclass classification; one versus all method was adopted whereby each subject or person was considered as one class and the total value is $k$ classes. Overall, the number of classifier used were $k(k-1) / 2$.For SVM, the testing was done with $15(15-1) / 2=105$ and $40(40-1) / 2=780$ classifier for Yale and ORL database, respectively. The data are divided into 50\% training and 50\% testing data by selecting the images randomly. RBF kernel function was used and 5 fold cross validation technique was implemented to obtain the best $C$ and $g$ parameter. The experimental setup consisted of two modes, i.e., user enrolment and recognition mode. The first mode procedure was that, a set of individuals in Yale/ORL database were used as an input to AMSR and the data later were train using SVM. The same procedures were implemented in the recognition mode which includes SVM testing process. The next experiment was done to get the effectiveness of the system using PCA as feature extraction method; the main reason is to understand the relevancy of PCA in the process. At the end of the experiments, the results using PCA method was compared with their own in which PCA achieved only $73.7 \%$ while the proposed technique accomplished $92 \%$ recognition rate.

\section{MATERIALS AND METHODS}

The biometrics presented in this research makes use of bi-modal, multi-algorithm techniques, as well as feature-level fusion. The design is bi-modal because both iris and face attributes are considered. The experiments are multi-instance because both right iris and left iris are used for iris matching. The experiments are multi-algorithm because two separate matching algorithms are used for the fused features.

Figure 1 presents the architecture of the system. The architecture comprises of modules that corresponds to the various processing stages of the system: image acquisition module, image detection module, image preprocessing module - which involves cropping and rescaling, segmentation or localization and normalization, feature extraction module, fusion module and classification module. The architecture also contains two biometrics subsystems each with an output Modality Accepted, $M_{A}$, or Modality Rejected $M_{R}$. 


\section{A. Facial and Iris Modality Acquisition Module}

Facial modality involves various steps such as face acquisition, detection, preprocessing, cropping and rescaling; and feature extraction. Facial images were acquired by using digital camera to capture a facial image $F_{i}$ of the dimension $w \times h$ (width $\mathrm{x}$ height) measured in pixels, from different subjects from the target audience - Religious groups. These acquired images were stored onto the database $D_{F_{i}}(r, c)$, a logically organized storage of facial image $F_{i}(w x h)$ pixelsin (.jpg)format extension file with varying sizes. Viola and Jones algorithm [17] is employed in this research to automatically detect face from an acquired image.

Iris modality involves an image acquirement, image preparation or pre-processing, attributes or feature extraction and normalization. In iris image acquisition, both left $E_{l}$ and right eye $E_{r}$ images were cropped from the preprocessed facial image $F_{i, C}(w \times$ $h$ ) of a particular subject and the cropped eyes $\left(E_{l, c} a n d E_{r, c}\right)$ were stored in the database $D_{E}(r, c)$ for pre-processing.

\section{B. Image Preprocessing Module}

\section{Facial Processing}

In Facial image pre-processing, the detected facial image $F_{i}(w x h)$ pixels is first cropped and resized before converted to grayscale. Then Histogram Equalization is applied to remove the lightening effect. The ROI (Region of Interest) portion is cropped first from the detected facial image, and then the rescaling is carried out by reducing original pixel of width $1920 \mathrm{X}$ height 1080 to $200 \times 220$ to aid memory requirement and computational efficiency.

This research makes use of luminosity method for the conversion of $F_{i}(w x h)$ pixels to grayscale. The triplet colour pixel (R, G, B) standing for red, green and Blue intensities in the range $[0,1]$ is used. Luminosity method of converting the given facial image to grayscale is presented in Equation 1. $F_{i, G s}=0.21 R+0.72 G+0.07 B$ (1) where $0.21,0.72$ and 0.07 are the eights of red, green and blue constituents in the colour image.

Equation 1 can be expressed mathematically as $A(x, y)=0.21 * \sum_{i=1}^{n} R(x, y)+0.72 *$ $\sum_{i=1}^{n} G(x, y)+0.07 * \sum_{i=1}^{n} B(x, y)$

Let $A(x, y)$ represent a pixel at a particular position in the colour image. Therefore $A(x, y, 1)$ is the value of the red pixel in $A(x, y)$, while $A(x, y, 2)$ gives the value of green pixel in $A(x, y)$, and $A(x, y, 3)$ represent the value of blue pixel in $A(x, y)$. In colourimetry, three numerical components are used to describe a colour.

The Histogram Equalization is applied on the graylevel facial image $F_{i, G s}$ in other to remove the lightening effect as presented in Equation 3.

Considering a graylevel facial image $F_{i, G s}$ denoted as a $m_{r}$ by $m_{c}$ matrix of integer pixel intensities extending from 0 to $L-1$, where the total number of pixels in an image is $n$, the number of pixels with graylevel is $n_{j}$ and the total number of graylevel that exist in the image, often 256 is $L$.

$F_{i, e q u l}=F_{i, G s}=\sum_{j=0}^{k} \frac{n_{j}}{n} k=0,1,2, \ldots, L-1$,

\section{Iris Preprocessing}

Segmentation of an iris image involves locating iris region $I_{r e g}$ in a cropped eye images $\left(E_{l, c}\right.$ and $\left.E_{r, c}\right)$. The iris region $I_{r e g}$ is estimated by dual circles, the iris/sclera region $I_{\text {reg }}$ is is one, while the other is located in the inner region to the first, the iris/pupil region $I_{r e g_{i / p}}$. Masek algorithm [9] is adopted and modified for segmentation of an iris in this research. It should be noted that, the left cropped eye image $E_{l, c}$ is used to describe the segmentation process, which as well applicable to the cropped right eye image $E_{r, c}$ to avoid repetition.

Hough transform is applied on the cropped left eye image $E_{l, c}$ to find the internal and external region of the iris and separate the iris radius from parameters defining the pupillary circle.

There is a generation of an edge map by computing the first derivatives of intensity values in a cropped left eye image $E_{l, c}$ and then finding the thresholding the magnitude of eye intensity gradient. $\mid \nabla G(x, y) \times$ $E_{l, c}(x, y) \mid(4)$

where $\nabla \equiv\left(\frac{\partial}{\partial \mathrm{x}}, \frac{\partial}{\partial \mathrm{y}}\right)$ is the derivative of the cropped left eye image $E_{l, c}$ in $x$ and $y$ direction and $G(x, y)=$ $\frac{1}{2 \pi \sigma^{2}} e^{\frac{\left(x-x_{0}\right)^{2}\left(y-y_{0}\right)^{2}}{2 \sigma^{2}}}$ is the Gaussian smoothing function with scaling parameter $\sigma$ selecting the proper scale of edge analysis. For the outer iris/sclera region $I_{\text {lreg }_{i / s}}$,gradients were biased in the vertical direction. Then, for the inner iris/pupil region $I_{\text {rreg }_{i / p}}$, vertical and horizontal gradients were weighted equally. 


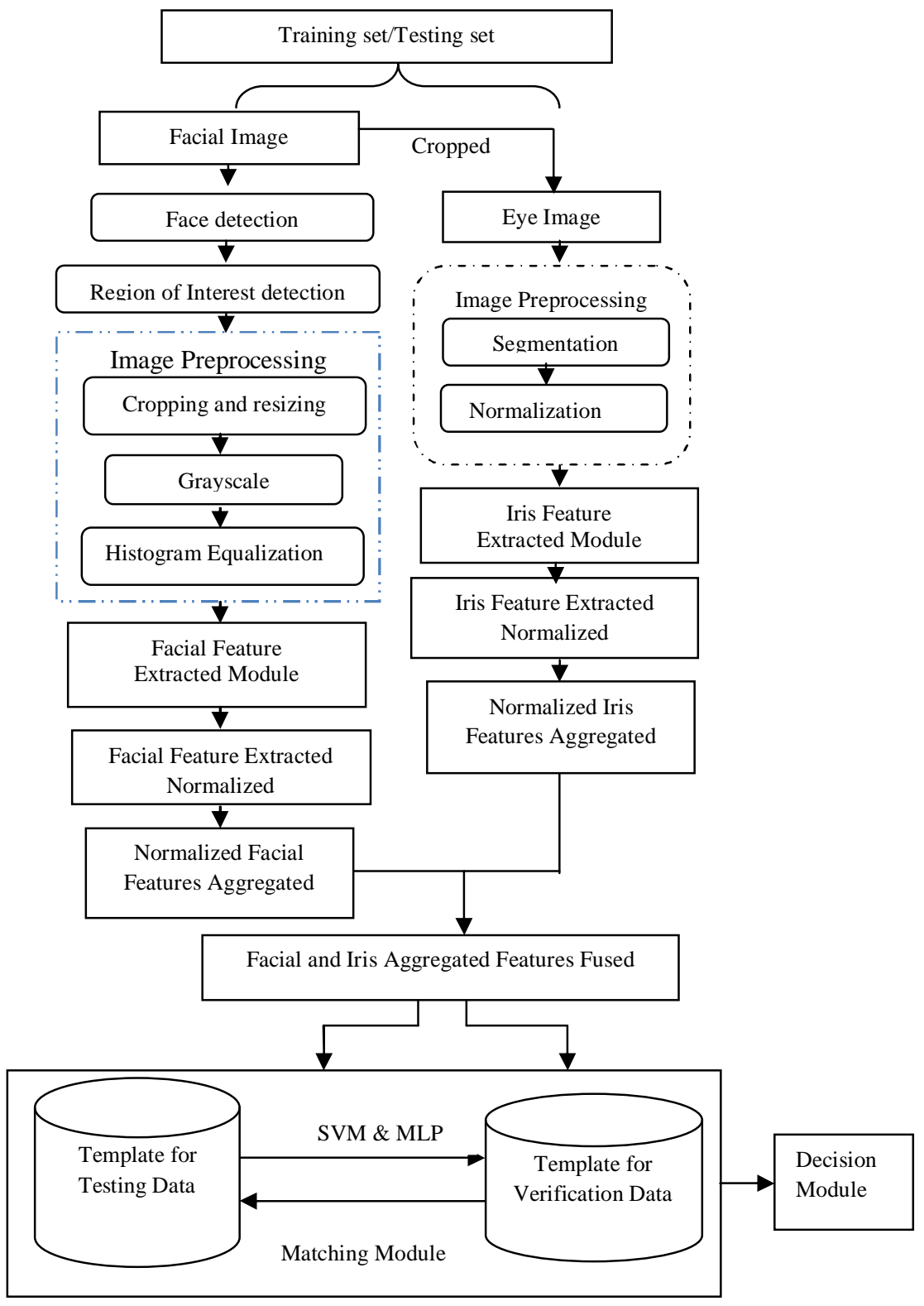

Figure 1: Architecture of Persons' Recognition System

Votes were casted in Hough space for the parameters of circles passing through each edge point from the edge map. The centre coordinates $x$ and $y$ and the radius $r$, which can help define any circle according to Equation 5 are these parameters:

$$
(x-a)^{2}+(y-b)^{2}-r^{2}=0
$$

This circle can be described by the two following equations:

$$
\begin{aligned}
& x=a+R \cos (\theta)(6) \\
& y=b+R \sin (\theta)(7)
\end{aligned}
$$

The points $(\mathrm{x}, \mathrm{y})$ trace the perimeter of a circle when the angle $\theta$ sweeps through the full 360 degree range. The radius and centre coordinates of the circle that best defined the edge points is the maximum point in the Hough space.

Then Circular Hough Transform with known radius $\mathrm{R}$ is applied on the cropped left eye image $E_{l, c}$ to detect the eyelids, thereby reducing the computational effect of the search, since $(a, b)$ the 
centre of the circle in $x$ and $y$ direction respectively only is searched for.

The Hough transform for the iris/sclera region $I_{\text {lreg }_{i / s}}$ is first carried out, the Hough transform for the iris/pupil region $I_{\operatorname{lreg}_{i / p}}$ is then carried out within the iris region $I_{\text {lreg }}$, instead of the entire eye region $E_{\text {lreg }}$, as the pupil is always within the iris region in order to make the circle detection procedure better and precise. The extracted features are hereby stored in the created iris database $D_{I}(r, c)$.

\section{Feature Extraction Module}

Feature extraction is the way of fetching out vital or significant structures from the preprocessed image. In this research facial features were extracted using three different methods namely: Gray Level Co- occurrence Matrix (GLCM) method, Principal Component Analysis (PCA) using the Singular Value Decomposition Techniques (SVD), and Gabor Filter method.

\section{a) Principal Component Analysis}

Principal component analysis (PCA) is a dimensionality reduction technique which is used for compression and face recognition problems. PCA calculates the eigenvectors of the covariance matrix, and projects the acquired data onto a lower dimensional feature space, which is defined by eigenvectors with large eigen values. Principal Component Analysis using Singular Value Decomposition algorithm is presented in Algorithm 1.

\section{Algorithm 1: Calculating PCs using SVD Method.}

1: $\quad$ Given a data matrix $\left(X=\left[x_{1} ; x_{2} ;:: ; x_{N}\right]\right)$, where $N$ represents the total number of samples and $x_{i}(M-$ 1) represents the $i^{\text {th }}$ sample.

2: $\quad$ The mean of all samples is computed as follows:

$$
\mu=\frac{1}{N} \sum_{i=1}^{N} x_{i}
$$

3: $\quad$ The mean is subtracted from all samples as follows:

$$
D=\left\{d_{1}, d_{2}, \ldots, d_{N}\right\}=\sum_{i=1}^{N} x_{i}-\mu
$$

4: $\quad$ The matrix is constructed as $Z=\frac{1}{\sqrt{N-1}} D^{T}, Z(N \times M)$

5: $\quad$ The SVD is calculated for $\mathrm{Z}$ matrix.

6: $\quad$ The square root of the sorted eigenvalues is the diagonal elements of $S$,

$\lambda=\operatorname{diag}\left(S^{2}\right)$, while the columns of $R$ represents the PCs.

7: $\quad$ The eigenvectors that have the largest eigenvalues $W=\left(R_{1} ; R_{2}, \ldots, R_{k}\right)$ is selected to construct the PCA space.

8: $\quad$ All samples are projected on the lower dimensional space of PCA $(W)$ as follows,

$$
Y=W^{T} D \text {. }
$$

\section{b) Gray Level Co-occurrence Matrix Method}

GLCM algorithm [19] works on grayscale image that has been quantized into different discrete gray levels. It is a $N \times N$ matrix, where $N$ is the number representing quantization level. The Algorithm 2 was employed in creating the matrix.

Algorithm 2: GLCM for image feature Extraction.

Create a GLCM $G$ with $N \times N$ size where $N$ is the quantization level of the input image

1: $\quad$ The size of the window for calculation will be $V$

2: $\quad$ The sample under consideration for the calculation will be $t$ 
3: $\quad$ Let $H$ be the sample set that surround $t$ that lie within a window centered on $t$ having its size specified under $V$

4: $\quad$ Considering only the samples in the set $H$, define each element $i, j$ of the GLCM to be the number of times two samples of intensities $i$ and $j$ occur in the sample $t$, where $i$ and $j$ are intensities between 0 and number of levels 1.

The sum of all elements $i, j$ of the GLCM is the total number of times the specified spatial relationship occur in $H$.

5: $\quad$ Convert the GLCM to a symmetric matrix by doing the following

i. $\quad$ Create transpose copy of the GLCM

ii. $\quad$ Add transpose copy to the original GLCM

6: $\quad$ Normalize the GLCM by dividing each element by the sum of all element

The element are now the probabilities of finding the relationship $i, j$ or $j, i$ in $H$.

7: $\quad$ Calculate the selected features using only the values in the GLCM

\section{c) Gabor Filter}

The one-dimensional Gabor [18] filter is defined as the multiplication of a cosine/sine (even/odd) wave with a Gaussian window. This is employed in this research for extracting salient features from face and iris modality as follows:

$g(x, y ; \lambda, \theta, \varphi, \sigma, \gamma)=$

$$
\begin{aligned}
& e^{\left(-\frac{x^{\prime 2}+\gamma^{2} y^{\prime 2}}{2 \sigma^{2}}\right) \cos \left(2 \pi \frac{x^{\prime}}{\lambda}+\varphi\right)}(8) g(x, y ; \lambda, \theta, \varphi, \sigma, \gamma)= \\
& e^{\left(-\frac{x^{\prime 2}+\gamma^{2} y^{\prime 2}}{2 \sigma^{2}}\right) \sin \left(2 \pi \frac{x^{\prime}}{\lambda}+\varphi\right)} \text { (9) }
\end{aligned}
$$

where $x^{\prime}=x \cos \theta+y \sin \theta$ and $y^{\prime}=-x \sin \theta+$ $y \cos \theta$.Lambda $(\lambda)$ is the wavelength parameters of the sinusoidal factor. $\lambda$ is the inverse of the frequency of the wave in the Gabor function with a value of $f=1 / \lambda$. Theta $(\theta)$ is the standard orientation of the parallel lines of Gabor function, its value is in degrees ranges amongst 0 and 360. $\mathrm{Phi}(\varphi)$ is the phase offset as a factor in the cosine Gabor function, its value in degrees ranges amongst -180 and 180 . Sigma $(\sigma)$ is the standard deviation of the Gaussian factor which decides the size of the (linear) support of the Gabor function. $\sigma$ value cannot be decides directly but can be transformed only through the value of the bandwidth $(b)$. Gamma $(\gamma)$ is the spatial aspect ratio that decides the shape of the ellipse of the Gabor function. Equation 8 and 9give the real and the imaginary components of Gabor functions. The parameters are adjusted accordingly to extract the salient features.

\section{Normalization and Feature Level Fusion of the Extracted Features}

Fusion achieves improved result if feature sets are the similar. We accomplished a Feature level fusion bybringing together the feature sets gotten from several information sources. But before concatenation of the features, there is normalization to convert the feature sets into a range that makes them more similar to each other.

Let $F=\left\{f_{1}, f_{2}, \ldots, f_{m}\right\}, I_{L f}=\left\{i_{l_{1}}, i_{l_{2}}, \ldots, i_{l_{n}}\right\}$ and $I_{R t}=\left\{i_{r_{1}}, i_{r_{2}}, \ldots, i_{r_{t}}\right\}$ denote feature vectors $\left(F \in \mathbb{R}^{m}, I_{L f} \in \mathbb{R}^{n}\right.$ and $I_{R t} \in \mathbb{R}^{t}$ ) presenting the information selected via three different sources Face, left Iris and right Iris respectively. The main goal is to bring together these feature sets in order to produce a new feature vector, $\mathrm{Z}$, in which individuals could be better represented. The features are then aggregated before fusion for enhanced presentation for the classifiers.

Min-max method is employed as follows: Let $f^{\prime}$ and $f$ denotes the feature set before and after normalization as follows

$$
f^{\prime}=\frac{f-\min (S)}{\max (\mathrm{S})-\min (S)}(10)
$$

where $f^{\prime}$ is the normalized feature set, $f$ is the input feature set, $S$ is the sum of inputs and $\min$ and $\max$ are the minimum and maximum feature set values that are produced by the matching algorithm being consider. Min-max mapped all feature sets into the range of 0.0 to 1.0 , but does not alter the shape of the features distribution. 
Normalizing the feature values based on these techniques generated modified feature vectors:-

$f^{\prime}=\left\{f_{1}^{\prime}, f^{\prime}{ }_{2}, \ldots, f_{m}^{\prime}\right\}, \quad I_{L f}^{\prime}=\left\{i_{l_{1}}^{\prime}, i_{l_{2}}^{\prime}, \ldots, i_{l_{n}}^{\prime}\right\}$ and

$I_{R t}^{\prime}=\left\{i_{r_{1}}^{\prime}, i_{r_{2}}^{\prime}, \ldots, i_{r_{Z}}^{\prime}\right\}$.

Due to the vast amount of features generated in this research data is aggregated by applying calculations across varying class, and then sum all the resulting summary information to present overall statistics. The normalized features (face, left iris and right iris) are aggregated as follows:

$f_{\text {agg }}=\sum_{i=1}^{N} f^{\prime}(i), \quad \forall i=1,2, \ldots, N(11)$

$I_{L_{a g g}}=\sum_{i=1}^{N} I_{L f}^{\prime}(i), \quad \forall i=1,2, \ldots, N(12)$

$I_{R_{\text {agg }}}=\sum_{i=1}^{N} I_{R t}^{\prime}(i), \quad \forall i=1,2, \ldots, N(13)$

where $N$ represent the number of samples, $i$ is the current sample being considered and $f^{\prime}, I_{L}^{\prime}$ and $I_{R}^{\prime}$ are the normalized feature dataset.

The aggregated feature vectors $f_{a g g}, I_{L_{a g g}}$ and $I_{R_{a g g}}$ is then combine to form $Z$.

$$
\begin{aligned}
& Z=
\end{aligned}
$$

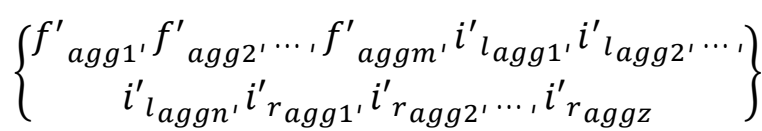

\section{E. Matching Methods}

The fused vector $Z_{i}$ for the training set and testing set were then presented as an input to the matchers SVM and Neural Network to classify as impostor and genuine sample. Comparison of recognition rate of individual classifier is then carried out according to the results generated.

For SVM, a kernel function is adopted to change the feature space into higher dimensional feature space in which the problem will become linearly separable because the dataset we are working on is of a nonlinear separable problem. The algorithm is presented as follows: each point $x$ in the input space is linked or connected to a point $z=\Phi(x)$ of a higher dimensional space, known as the feature space, where the data are set apart from each other by a hyperplane [11]. The major points here is that the mapping $\Phi($. )must meet the criteria in which the dot product of two points in the feature space $\Phi(x) \cdot \Phi(\mathrm{y})$ can be written anew as a Polynomial kernel function $K(x, y) \cdot L_{D}(w, b, \alpha)=$ $\sum_{i}^{N} \alpha_{i}-\frac{1}{2} \sum_{i=1}^{N} \sum_{i=1}^{N} \alpha_{i} \alpha_{j} y_{i} y_{j} K\left\langle x_{i} \cdot x_{j}\right\rangle(15)$

subject to

$0 \leq \alpha_{i} \leq C$

and

$\sum_{i=1}^{N} \propto_{i} y_{i}=0$

where $K$ is the polynomial kernel function.

For MLP classification, a back propagation learning algorithm applied for updating of weight and error correction which involves four steps is given as follows:

\section{Algorithm 3: Back propagation Learning Algorithm}

STEP 1: Initialization

The entire weights and threshold levels of the network were fixed to a random numbers evenly distributed inside a small range. The weight initialization is carried out on a neuron -by - neuron basis.

STEP 2: Activation

Activation of the back propagation neural network is done by applying inputs $z_{1}(P), z_{2}(P), \ldots z_{n}(P)$ and desired output $y_{d, 1}(P), y_{d, 2}(P), \ldots y_{d, n}(P)$.

a) Calculations of the actual outputs of the neurons in the hidden layer were done;

$$
Y_{j}(P)=\operatorname{sigmoid}\left[\sum_{i=1}^{n} z_{i}(P) \times w_{i j}(P)-\theta_{j}\right]
$$

where $\mathrm{n}$ is the number of inputs of neuron $\mathrm{j}$ in the hidden layer, and sigmoid is the activation function.

b) Calculations of the actual outputs of the neurons in the output layer were carried out as;

$$
Y_{k}(P)=\text { sigmoid }\left[\sum_{j=1}^{m} z_{j k}(P) \times w_{j k}(P)-\theta_{k}\right]
$$


where $\mathrm{m}$ is the number of inputs of neuron $\mathrm{k}$ in the output layer.

STEP 3: Weight Training

Updatingof the weight in the back propagation network by propagating the errors associated with output neurons is done.

a) calculation of the error gradient for the neurons in the output layer is carried out as:

$$
\delta_{k}(P)=Y_{k}(P) \times\left[1-Y_{k}(P)\right] \times e_{k}(P)
$$

where

$$
e_{k}(P)=Y_{d, k}(P)-Y_{k}(P)
$$

To calculate weight corrections:

$$
\Delta w_{j k}(P)=\alpha \times Y_{j}(P) \times \delta_{k}(P)
$$

where $\alpha$ is the learning rate,

Then, we update the weight at the output neuron

$$
w_{j k}(P+1)=w_{j k}(P)+\Delta w_{j k}(P)
$$

b) calculation of the error gradient for the neurons in the hidden layer is done thus;

$$
\delta_{j}(P)=Y_{j}(P) \times\left[1-Y_{j}(P)\right] \times \sum_{k=1}^{l} \delta_{k}(P) \times w_{j k}(P)
$$

To calculate the weight corrections:

$$
\Delta w_{i j}(P)=\alpha \times x_{i}(P) \times \delta_{j}(P)
$$

The weights at the hidden neurons were then updated as;

$$
w_{i j}(P+1)=w_{i j}(P)+\Delta w_{i j}(P)
$$

STEP 4: Iteration

Iteration $\mathrm{P}$ is increased by one, then go back to step 2 and repeat the process until the selected error criterion is satisfied.

\section{RESULT DISCUSSION}

Some of the results of various modules in the architecture are presented as follows:
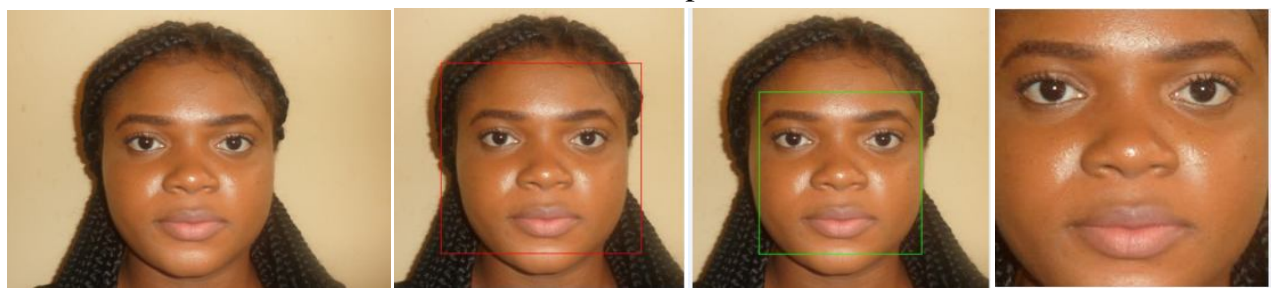

Figure 2: Acquired, Detected, ROI and Cropped of Sample Facial Image
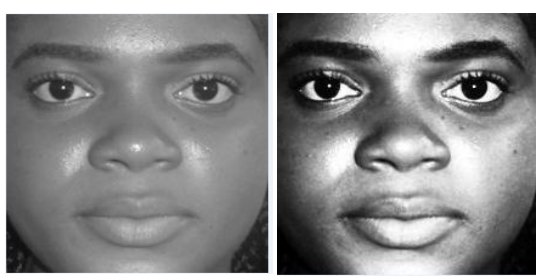

Figure 3: Conversion to Grayscale, Histogram Equalized of Sample Face

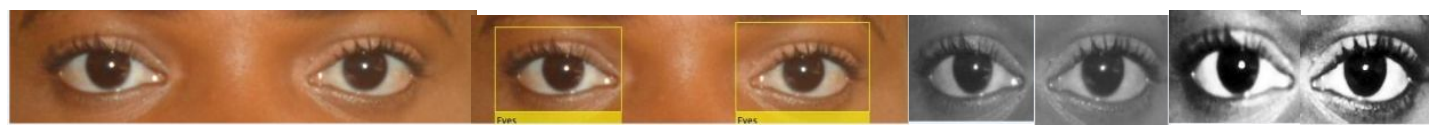

Figure 4: Cropped Eyes, Detected Eyes, Grayscale and Histogram Right and Left Eyes 


\section{a) Singular Value Decomposition Method} Images of 200 X 220 dimensions are passed into PCA using SVD method. Diagonalizing the data matrix $\left(X \in R^{p \times q}\right)$ into three matrices $X=L S R^{T}$ is the aim, where $L(p \times p)$ are known as left singular vectors, $S(p \times q)$ is a diagonal matrix signifies the singular values that are arranged from high-to-low, and $R(q \times q)$ represents the right singular vectors. The columns of the right singular vectors $(R)$ signify the eigenvectors of $X^{T} X$ or the principal components of the PCA space, and $S_{i}^{2}, \forall i=1,2,3 \ldots . . q$ represent their corresponding eigenvalues. Figure 5 presents the sample data of the SVD

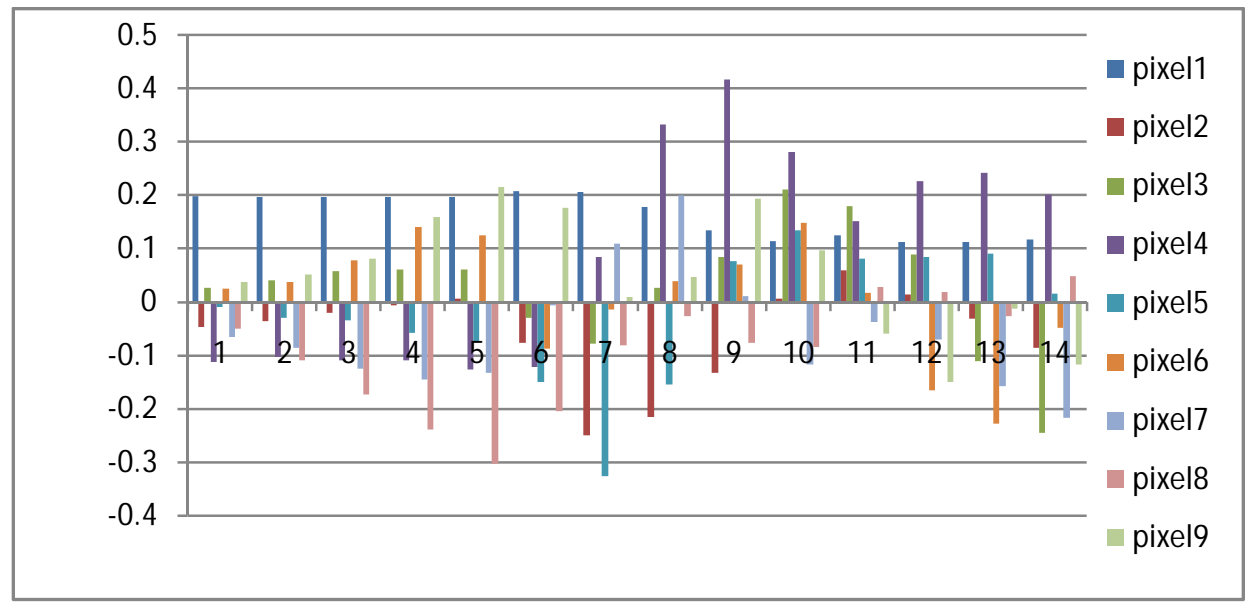

Figure 5: Eigenvectors or Principal Components Sample Data Chart

\section{b) Gabor Filter Method}

Feature image is extracted using a Gabor filter in accordance with the selected adaptive orientation, size, frequency, and segment for each pixel. The sample data of the extracted features is shown in Figure 6.

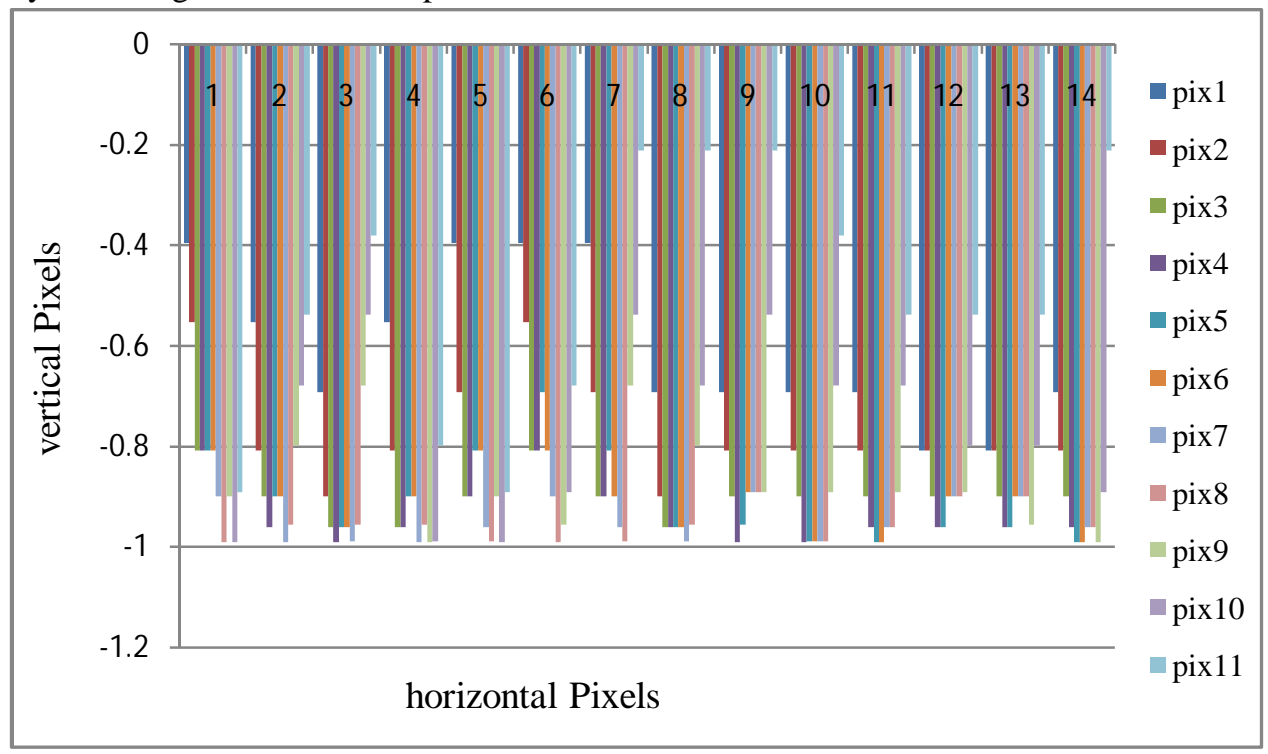

Figure 6: Sample data Chart of Extracted features of an image using Gabor Filter

\section{A. Performance Metrics of KNN, SVM and ANN Classification Models}

This was carried out using True Positive Rate (TPR), False Positive Rate (FPR), Confusion Matrix and
Accuracy. The template matching was trained using 250 dataset. The trained model was tested with 37 dataset of unknown classification and validated with 38 dataset. The model correctly classified 150 as 
genuine persons meaning that the True Positive Rate is $97 \%$ for class 1 and 5 as impostor meaning the False Positive Rate is 3\%, the remaining 20 dataset for class 0 were correctly classified as impostor having $100 \%$ correct prediction. The results are shown in Figure 7.

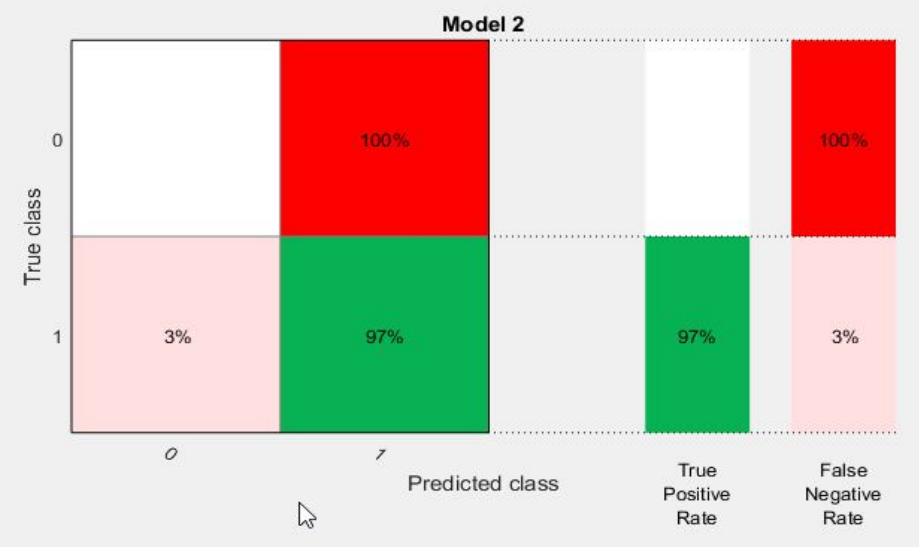

Figure 7: TPR and FNR for trained SVD data using Template Matching

A multiclass SVM classifier was used to carry out a person recognition rate for the algorithm. 250 dataset were used with $70 \%$ as training set, the remaining $15 \%$ for testing and $15 \%$ as validation. The data is divided into two classes; 1 which represents the genuine and 0 which represents the impostor. True positive rate for class 1 is $100 \%$ while that of class 0 is $0 \%$. False Positive Rate for class 1 is $0 \%$ while for class 0 is $100 \%$. The result is shown in Figure 8.

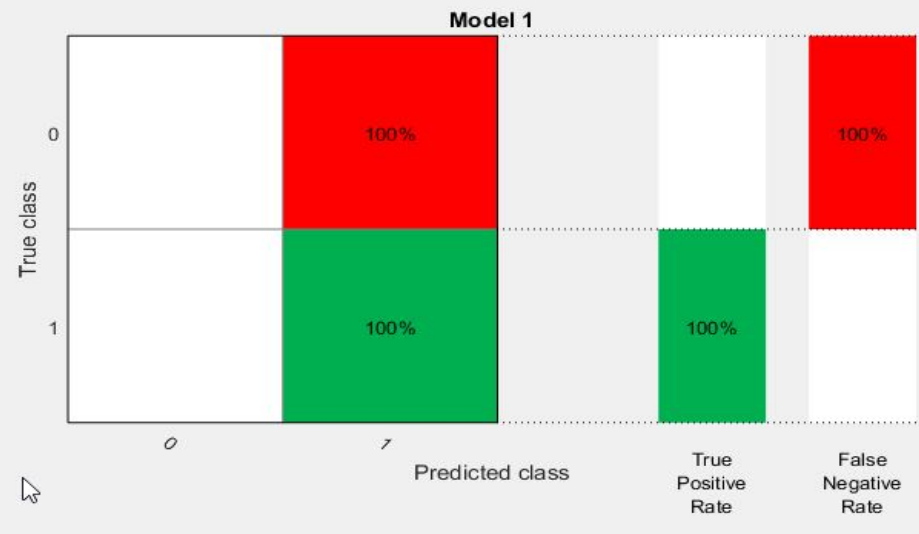

Figure 8: True Positive Rate and False Negative Rate of Class 1 and 0 using SVM.

The three classifiers are evaluated as follows:

SVM and ANN correctly classified faces better than

KNN. Gabor dataset has the overall best performance. The charts are presented in Figure 9 and 10 . 


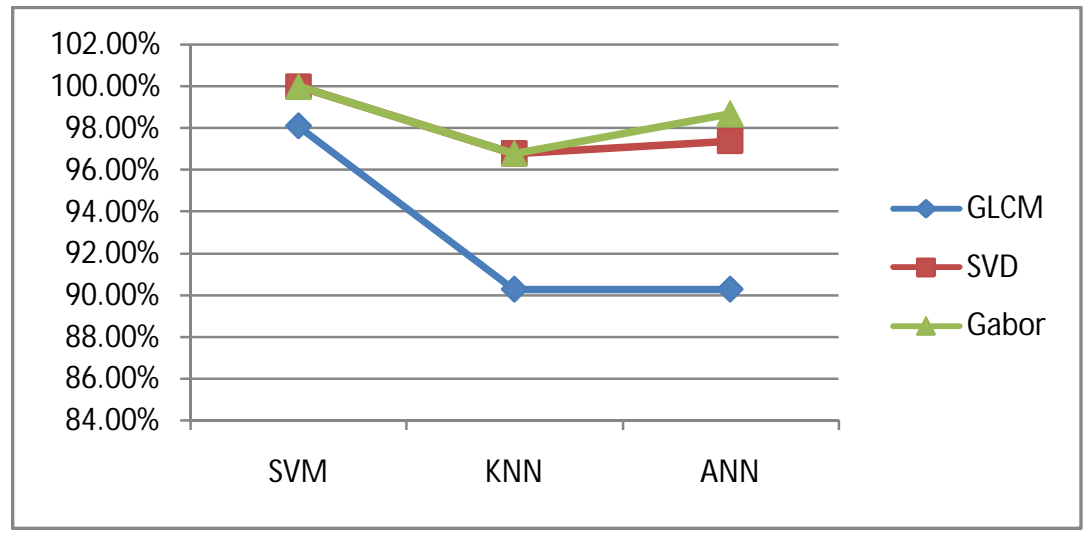

Figure 9: True Positive Rate of the Three Classifiers

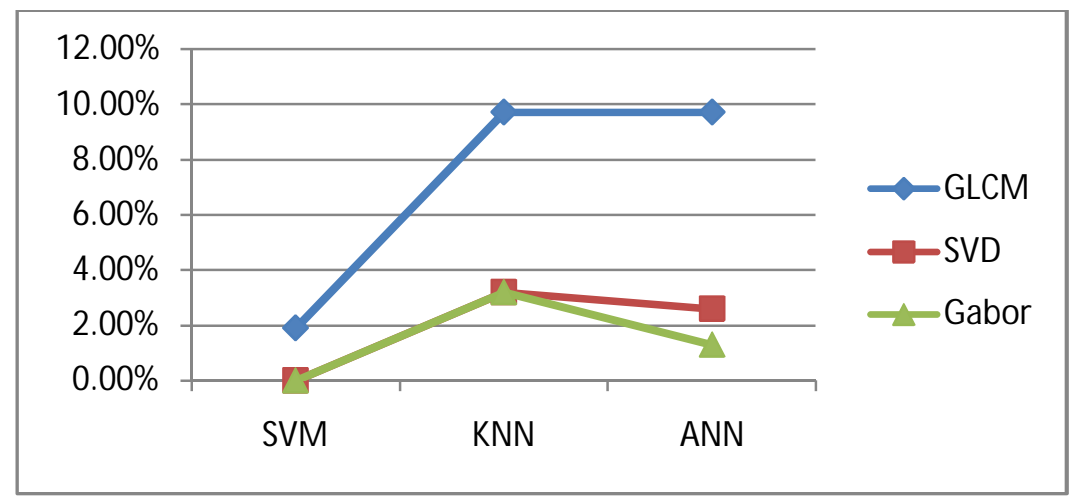

Figure 10: False Positive Rate of the Three Classifiers

\section{CONCLUSION}

In this research, features of the facial and iris modalities were extracted using three different feature extraction methods: GLCM, SVD and Gabor filter. The extracted features were conbined together employing aggregation method. Classification was done using ANN and SVM and the performance evaluation was carried out using KNN. Gabor filtered extracted features performs better with SVM as classifier, where all persons were classified correctly. ANN performs well on SVD filtered extracted features where only four persons were misclassified and KNN performs well on Gabor filtered extracted features, there was a misclassification of five persons.

At the end of the comparative analysis, it was discovered that the SVM has the better performance on all filtered extracted features with the result of 92.6\% on GLCM, $93.3 \%$ on SVD and $94.0 \%$ on Gabor filtered extracted features. ANN performed better also with result of $90.0 \%$ on GLCM, $92.7 \%$ on SVD and $92.3 \%$ on Gabor filtered extracted features.

\section{REFERENCES}

[1] H.Abdenour, Learning and Recognizing Faces: from still images to video sequences, Academic dissertation presented with the assent of the faculty of technology, University of Oulu, 2005.

[2] R. D.Chaudhari, Artificial intelligence plays an important role using various sensors for identification of person's iris, International Journal of Basic and Applied Research Special Issue, 2012,(95-97).

[3] J.Daugman, Uncertainty relation for resolution in space, spatial frequency, and orientation optimized by twodimensional visual cortical filters,'J. Opt. Soc. Amer. A, 2(7):1985, 1160-1169.

[4] A. K.Jain, A.Ross, and S.Pankanti,Biometric: A Tool for Information Security. IEEE Trans, Information Forensics and Security, 1(2): 2006,125-144.

[5] M.Jan, M.Martin, O.Milo's and P.Jarmila, Support Vector Machines, PCA and LDA in Face Recognition,Journal of Electrical Engineering, 59(4): 2008,203-209.

[6] B.Jayanta, K.Kiran, T.VivekandR.Nalini,QPLC: A Novel Multimodal Biometric Score Fusion Method, 
International Journal of Biometrics and Bioinformatics (IJBB), 6(5): 2012,123-134.

[7] D.Jobson, Z.Rahman and G. A.Woodell, A multiscaleretinex for bridging the gap between color images and the human observation of scenes, IEEE Trans. Image Proc., Vol6:1997, 965-976.

[8] P. A.Johnson, F.Hua, and S.Schuckers, Comparison of Quality-Based Fusion of Face and Iris Biometrics, (2011 IEEE.

[9] M. Libor, Recognition of Human Iris Patterns for Biometric Identification, BSc. Report in School of Computer Science and Software Engineering, The University of Western Australia (2003).

[10] K. A.Olatunji,Development of Machine Learning Algorithm for Face and Iris Recognition, A thesis in the Department of Computer Science, School of Computing, submitted to the School of Postgraduate Studies, in partial fulfilment for the requirements for the award of Doctor of Philosophy in Computer Science of The Federal University of Technology, Akure, Nigeria (2019).

[11] K. A.Olatunji, A.Oguntimilehin and O. A.Adetunmbi, Bi-Modal Methods- An Overview, International Journal of Basic and Applied Science (IJBAS), EISSN: 2301-4458; 2301-8038:(2015) 44-53

[12] A.Ross and A. K.Jain, Information fusion in biometrics,Pattern Recognition Letters, 24: (2003) 2115-2125.

[13] C.Sanderson andK. K.Paliwal, Information fusion and person verification using speech and face information, Research Paper IDIAP-RR 02-33, IDIAP, September 2002.

[14] M. M.Sani, K. A.Ishak and S. A.Samad, Classification using Adaptive MultiscaleRetinex and Support Vector Machine for Face Recognition System,Journal of Applied Sciences, 10: (2010) 506-511.

[15] R.GanorkarSanjay, and A. Ghatol,Ashok,Iris Recognition: An Emerging Biometric Technology, In Proc. of the 6th WSEAS International Conference on Signal Processing, Robotics and Automation, Greece, Feb. 2007, 91 - 96.

[16] T.Upasana,K.Deepali and T.Abhishek, IRIS recognition based on PCA based Dimensionality reduction and SVM,International Journal of Computer Applications, 49(3): (2012) 28-32.

[17] P.Viola and M. J.Jones, Robust Real-time Face Detection, International Journal of Computer Vision.

57: (2004) 137-154

[18] D.Gabor, Theory of communication, Journal of the Institute of Electrical Engineers, 93(1946): 429-457.

[19] H. Mryka, Practical Guidelines for Choosing GLCM textures to use in Landscape Classification tasks over a Range of Moderate Spatial Scales, International Journal of Remote Sensing, 38(2017): 1312-1338. 